\title{
Design of Compact Stacked-Patch Antennas on LTCC Technology for Wireless Communication Applications
}

\author{
R. L. Li, K. Lim, M. Maeng, E. Tsai, G. DeJean, M. Tentzeris, and J. Laskar \\ School of Electrical and Computer Engineering \\ Georgia Institute of Technology, Atlanta, GA 30332-0250, USA
}

\section{Introduction}

The exploding growth of wireless communications systems leads to an increasing demand for integrated compact low-cost antennas. As a result, LTCC (Low Temperature Co-fired Ceramics) multilayer technology is becoming more and more popular for the production of highly integrated, complex multilayer modules and antennas. This technology is appreciated for its flexibility in realizing an arbitrary number of layers. However, LTCC materials typically possess a high dielectric constant. On one hand, this helps reduce the antenna size, but on the other hand also reduces the bandwidth of planar patch antennas, thus making the design for practical wireless communication systems more difficult and therefore more challenging.

A common approach for improving the bandwidth performance of a patch antenna is to add parasitic elements to the antenna structure, e.g., a stacked patch [1]. This reduces the impedance variation of the antenna with frequency, thus enhancing bandwidth performance. Various arrangements of stacked structures have been investigated in [2][3]. Typically a foam and low dielectric constant substrate stacked configuration or a high and low dielectric constant material combination is used to achieve a broad bandwidth. These structures require a thicker substrate (typically the total antenna thickness $=\sim 0.1 \lambda_{0}$, $\lambda_{0}=$ wavelength in free space) and different materials. This is not suitable for the compact antenna requirement and LTCC technology. In addition a tedious procedure may be required in the design of such a different-substrate-stacked patch antenna. There are many parameters that need to be adjusted for an optimized bandwidth performance, such as the length and width of each patch, the thickness of each substrate, as well as the position of the feed point. In practice it is very difficult to adjust every parameter for optimal performance.

In this paper, a simple design method is presented for compact stacked-patch antennas based on LTCC multi-layer structures. It is found that an optimal bandwidth performance always can be obtained by changing only the number of the LTCC layers. The design methodology is presented first followed by its applications to three emerging wireless communication systems. It will be shown that the stacked-patch antenna on LTCC multilayers can achieve a bandwidth of as much as twice of that for a single-patch antenna with the same substrate. Such a bandwidth may meet the requirements of several wireless communication systems, such as Bluetooth, IEEE 802.11a, as well as the broadband LMDS (Local Multi-point Distribution System).

\section{Antenna Structure and Design Strategy}

The antenna structure considered throughout this paper is shown in Fig. 1. Two square patches (lower and upper) have the same dimensions and are stacked on a grounded LTCC substrate with a total thickness of $h$. The lower patch divides the total substrate into a lower substrate (thickness $=h_{1}$ ) and an upper substrate (thickness $=h_{2}$ ). The stacked patch is backed by a metal cavity while the lower patch is fed through a probe extended 
from a $50 \Omega$ coaxial line. In modern wireless communication systems, it is desirable to integrate the antenna with other RF circuits. Therefore it is essential to prevent any unwanted radiation from other RF components in the integrated chip. This is the reason we introduced a metal-backed cavity in order to shield the RF signals generated inside the chip from the antenna signals. In LTCC technology, the side wall of the metal cavity can be easily realized through an array of vertical vias. We select the lateral dimension of the cavity to be twice of that of the stacked patch to avoid taking into account the effect of the side wall on the impedance characteristic of the antenna.

The LTCC multilayer structure used in our investigation consists of LTCC GL550 ceramic material of equal layer thickness ( 4 mils). The dielectric constant $\varepsilon_{\mathrm{r}}$ of the LTCC substrate equals 5.6 and the loss tangent is 0.0012 .

We investigated the impedance performance of the stacked-patch antenna through numerous numerical simulations. It was found that the position of the feed probe must be fixed at the center of the edge of the lower patch in order to match with a $50 \Omega$ coaxial line. We also have realized that it is possible to achieve a maximum bandwidth only by the adjustment of the thickness of the upper substrate while keeping the sizes of the lower substrate and stacked patch unchanging. This feature is suitable for LTCC mutlilayer structures and is very helpful in simplifying the design procedure for practical broadband stacked-patch antennas. In the wake of many practical experiences, we summarize the basic steps of the stacked-patch antenna design as follows.

(1) Select the preliminary total thickness $h\left(=h_{1}+h_{2}\right)$ of substrate according to the bandwidth requirement for a specific application. We have investigated the relationship between the bandwidth and substrate thickness for a compact substrate (thickness $=0.01-0.03 \lambda_{0}$ ), which is shown in Fig. 2, where the relative 10-dB returnloss bandwidth (normalized to the center frequency $f_{\mathrm{c}}$ ) is plotted as a function of the thickness (normalized to the free-space wavelength $\lambda_{0}$ at $f_{\mathrm{c}}$ ). For comparison the bandwidth of a traditional single-patch antenna with the same type of substrate is also presented in this figure. It is observed that the compact stacked-patch antenna can achieve a bandwidth of up to $7 \%, 60 \%-70 \%$ wider than a sing-patch antenna could.

(2) Select the thickness of lower substrate $h_{l}$. In order to achieve a broadband performance it is essential to form a resonant loop in a Smith chart of the input impedance. We have found through numerous simulations that for the LTCC substrate the resonant loop will appear when the ratio of the lower-substrate thickness to that of the upper substrate is around 1:3. Therefore we can choose $h_{l}=h / 4$.

(3) Design initially the length of the stacked patch $L$ according to the specified center frequency for a practical application. For convenience, we suggest making use of a typical approximate equation [1]: $L=1 / 2 f_{c} \sqrt{\mu_{0} \varepsilon_{0} \varepsilon_{r}}$.

(4) Determine the upper-substrate thickness $h_{2}$ for an optimal return loss. The initial value of $h_{2}$ can be chosen as $3 h_{1}$ according to Step (2) The final value of $h_{2}$ may be obtained by numerical simulation. Upon our observation, it is found that the resonant loop in the Smith chart will move from the upper Smith chart (inductive) to the lower (capacitive) as the upper patch moves toward the lower patch. $h_{2}$ is determined when the center of the resonant loop moves most close to the center of the Smith chart, which corresponds to a minimum return loss.

(5) Adjust $L$ exactly to make the bandwidth fully cover the specified band of the practical specification. This also can be carried out with the help of numerical simulation.

If the final optimized bandwidth does not meet the requirement for the specified band, we have to increase $h_{1}$ a little bit and repeat Steps (4) and (5) until the specification is satisfied. 


\section{Application}

As an example, we now apply the design principles described above to the antenna design for LMDS $28 \mathrm{GHz}$ band $(27.5-29.25 \mathrm{GHz})$ [4]. The simulated results presented here are obtained by using a TLM-based software: Micro-Stripes 5.6, and validated by an FDTD solver.

According the band specification, the relative bandwidth is calculated to be $6.2 \%$. Looking up Fig. 2, we find the total thickness of substrate to be about $0.0275 \lambda_{0}$. The center frequency $f_{c}$ of this band is $28.375 \mathrm{GHz}$. Therefore the physical thickness of the substrate is $0.29 \mathrm{~mm}$, which is approximately equal to 3 LTCC layers. Considering that the lower-substrate thickness should be roughly a quarter of the total substrate thickness, we thus choose $h_{1}=1$ layer. Substituting $f_{c}=28.375 \mathrm{GHz}$ into (1), we get $L=88$ mils. Now we began to search for the upper-substrate thickness $h_{2}$ through numerical simulation. The simulated input impedance is plotted for different $h_{2}$ (in layers) in a Smith chart as illustrated in Fig. 3. We can see that the resonant loop does move from the upper to lower Smith chart as $h_{2}$ reduces. The optimal bandwidth performance is obtained when the resonant loop is completely located inside the 10-dB return loss circle. We find $h_{2}=2$ layers, which is one layer fewer than the initial value of $h_{2}$. Next $L$ needs to be adjusted to meet the band specification. Upon simulation, $L$ needs to be reduced by $L=80$ mils. The final result for the impedance characteristic is depicted in Fig. 4. To demonstrate the validity of the simulated result, the return loss calculated by using FDTD is also presented in this figure. Obviously the two simulated results agree well, both cover the specified band.

The radiation pattern of the stacked-patch antenna is very similar to that for a typical single-patch antenna. Fig. 5 shows a pattern comparison between stacked- and singlepatch antennas. We can see that the co-polarized components are completely same while the cross-polarized component for the stacked patch is 10-dB lower than that for the single patch due to a much shorter fed probe for the stacked patch.

\section{Conclusion}

Stacked-patch antennas on LTCC multiplayer structures can be easily designed by only an adjustment of the number of LTCC layers. The methodology presented in this paper is suitable for the design of compact broadband antennas. It is demonstrated that a stackedpatch antenna on an LTCC substrate with a thickness of less than $0.03 \lambda_{0}$ can achieve a bandwidth of up to 7\%, which may find applications in a number of wireless communication systems.

\section{Acknowledgement}

The authors wish to acknowledge the support of Yamacraw Design Center, the NSF Career Award, and the NSF Packaging Research Center.

\section{References}

[1] P. Bhartia, Inder. Bahi, R. Garg, and A. Ittipiboon, Microstrip Antenna Design Book, Artech House, 2000.

[2] R. B. Waterhouse, "Design of probe-fed stacked patches," IEEE Trans. Antennas Propagat. vol. 47, no. 12, pp. 1780-1784, Dec. 1999.

[3] R. B. Waterhouse, "Stacked patches using high and low dielectric constant material combinations," IEEE Trans. Antennas Propagat. vol.47, no.12, pp. 1767-1771, Dec. 1999.

[4] A. Nordbotten, "LMDS systems and their applications," IEEE Communications Magazine, vol. 38 , no. 6, pp. 150-154, June 2000. 


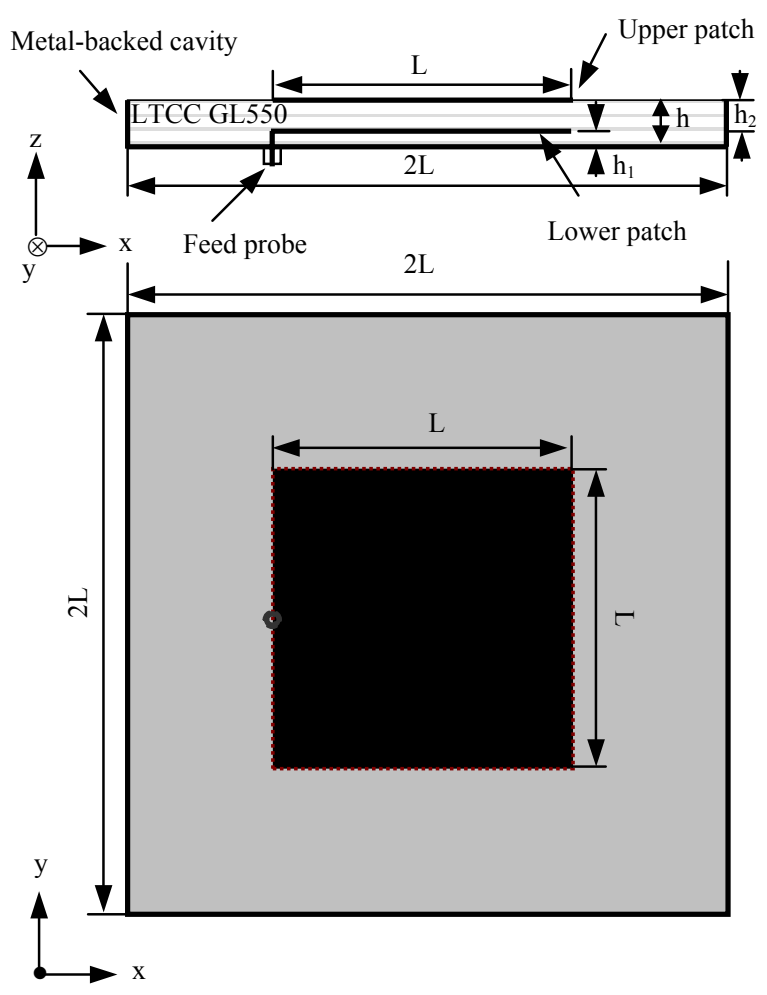

Fig. 1. Probe-fed cavity-backed stackedpatch antenna on LTCC multilayer substrate.

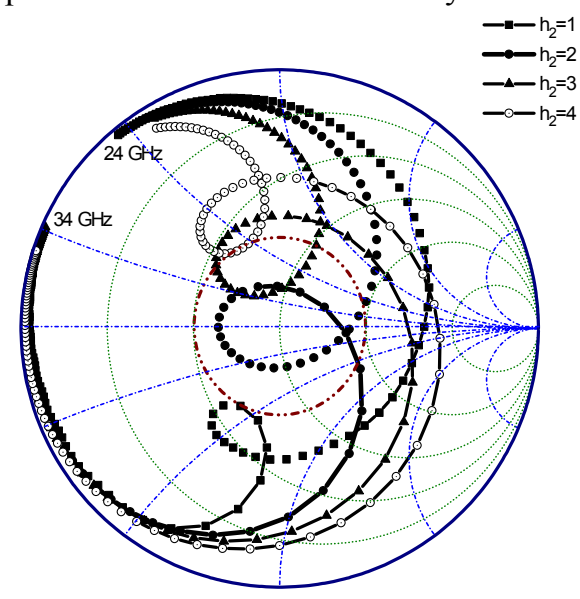

Fig. 3. Effect of $h_{2}$ on stacked-patch antenna design for LMDS band.

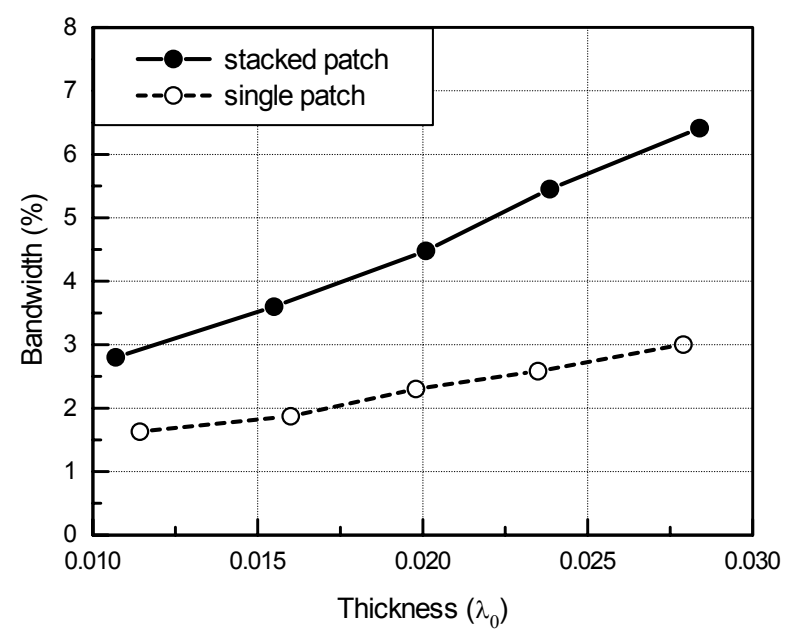

Fig. 2. Bandwidth performances of stacked- and single-patch antennas as a function of their substrate thickness.

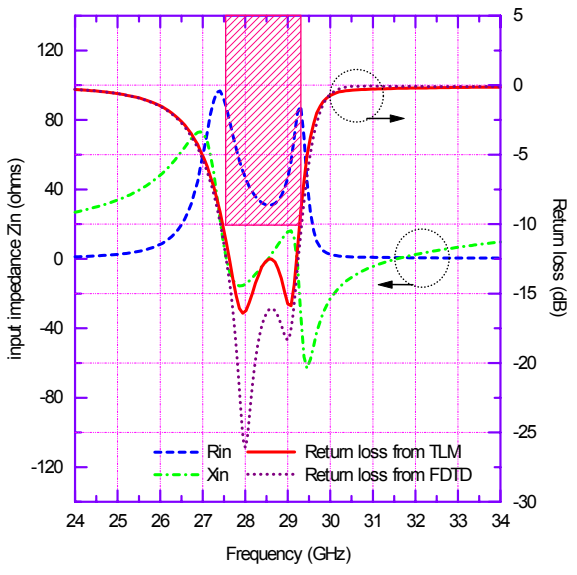

Fig. 4. Input impedance characteristic of stacked-patch antenna for LMDS band.

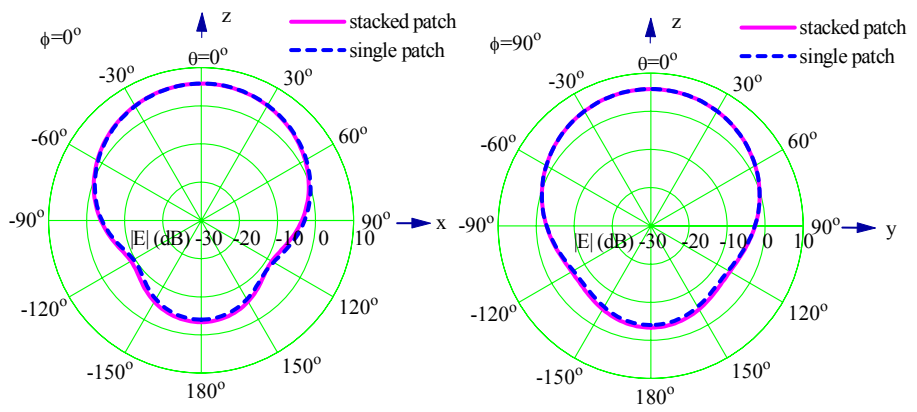

(a) co-polarized component

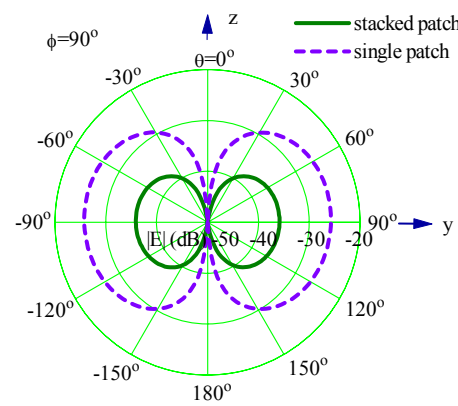

(b) cross-polarized component

Fig. 5. Pattern comparison of stacked- and single-patch antennas for LMDS band at $28 \mathrm{GHz}$. 